\title{
Time-resolved Study of Light-induced Ground State Proton Transfer from Acid Medium to 4-nitrophenolate
}

\author{
Leandro Scorsin ${ }^{1}$, Letícia S. Martins ${ }^{2}$, Haidi D. Fiedler ${ }^{1}$, Faruk Nome $^{1}$, René A. Nome², \\ ${ }^{1}$ Department of Chemistry, National Institute of Catalysis, Federal University of Santa Catarina (UFSC), \\ Florianópolis, SC 88040-900, Brazil. \\ ${ }^{2}$ Institute of Chemistry, State University of Campinas (UNICAMP), Campinas, SP 13083-970, Brazil. \\ Correspondence
}

Leandro Scorsin, Department of Chemistry, National Institute of Catalysis, Federal University of Santa Catarina, Florianópolis, SC 88040-900, Brazil. E-mail: lpscorsin@gmail.com

René A. Nome, Institute of Chemistry, State University of Campinas, Campinas, SP 13083-970, Brazil. E-mail: nome@unicamp.br

\begin{abstract}
In the present work, we study the transient laser-induced formation of 4-nitrophenolate (4-NPO') in the ground electronic state and subsequent proton transfer reaction with acetic acid and water with numerical calculations and laser flash photolysis. We employ the Debye-Smoluchowski sphericallysymmetric diffusion model of photoacid proton transfer to determine experimental conditions for studying thermally activated chemical reactions in the ground electronic state. Numerically calculated protonation and deprotonation probabilities for 4-NPO ${ }^{-}$and 4-nitrophenol (4-NPOH) in both ground and excited states showed the feasibility of efficiently producing the ground state anion in the photoacid cycle. We performed laser flash photolysis measurements of 4-NPOH to characterize the photo-initiated ground state protonation and deprotonation rate constants of 4-NPO$/ 4-\mathrm{NPOH}$ as a function of acetic acid, $\mathrm{pH}$, temperature and viscosity. Overall, the work presented here shows a simple way to study fast competing bimolecular proton transfer reactions in non-equilibrium conditions in the ground electronic state $(G S P T)$.
\end{abstract}

KEYWORDS: Ground State Proton Transfer, Spherically-Symmetric Diffusion Model, DebyeSmoluchowski Diffusion Model, Laser Flash Photolysis, 4-nitrophenol/4-nitrophenolate 


\section{1 | INTRODUCTION}

Many chemical reactions in natural and synthetic systems are thermal processes that occur in the ground electronic state. Mechanistic studies of such processes to determine elementary reaction steps are usually performed within the conceptual framework of chemical kinetics, typically with up to microsecond timescale resolution. ${ }^{1}$ In order to study chemical reactions at shorter timescales, stroboscopic tools have been developed to characterize molecular motions and chemical reactions in the nanosecond to femtosecond range. ${ }^{2}$ For example, in ultrafast transient absorption spectroscopy, pump light absorption induces a macroscopic polarization in the sample, thereby synchronizing an ensemble of individual molecules; the subsequent dynamics is then studied with the probe light. ${ }^{3}$ Such pumpinduced synchronization of molecular dynamics is crucial for investigating chemical reactions with high time resolution, and it has enabled the study of photo-induced reaction mechanisms with remarkable detail.

Given the wide occurrence of thermal ground-state reactions in Chemistry, we hope it is useful to develop novel experimental approaches enabling the study of asynchronous processes with similar time resolution and level of detail as provided by synchronous/stroboscopic laser-based techniques. ${ }^{4,5}$ Near equilibrium, the application of perturbation-based approaches to study molecular mechanisms of thermal processes is based on the fluctuation-dissipation theorem. For example, the correlation-function formalism of molecular spectroscopy has been applied to obtain dynamical information from spectroscopic tools such as nuclear magnetic resonance and vibrational (infrared and Raman) spectroscopies.

Although this approach is in principle valid for any thermal process in the linear response regime, the application of stroboscopic approaches to study chemical reactions on slower timescales - such as reactions driven by thermal fluctuations on the microsecond-to-hours timescale - is less common. ${ }^{2}$ In order to study thermal reactions in the ground state and with higher time resolution (picoseconds to microseconds), we have researched a chemical system absorbing short laser pulse excitation light and 
efficiently leading to the formation of a new chemical species in the ground state. ${ }^{6,7}$ Based on this system preparation approach, the new species will be in a non-equilibrium concentration induced by the pump pulse.

We report numerical investigations and nanosecond transient absorption studies on 4-nitrophenol (4-NPOH). Past studies with phenols have been widely analyzed for its electronic properties and structural variations. ${ }^{8-12}$ 4-NPOH has been used as leaving group in many kinetics studies accompanied by UV-vis spectroscopy in reactions that span from milliseconds to hours, because it can be conveniently detected by UV-Vis spectroscopy. ${ }^{18,19}$ Techniques using flash photolysis with scanning in the UV-Vis or infrared spectrum also make it possible to follow the formation of transient species. ${ }^{13-15}$ Experimental characterization of $p K_{a}$ changes or solvation medium, imply kinetic and thermodynamic changes for this proton transfer reaction in ground and excited states. Reactions involving nitrophenol degradation were also performed on suspensions, or colloidal dispersions supported on surfaces of oxides of metals such as titanium and iron. ${ }^{16,17}$

In the present work, 4-NPOH was selected because phenols are efficient photoacids, a fact that allows proton transfer to water (or an acceptor) and formation of nitrophenolate in the nanosecond range by a mechanism of protolysis and not by direct proton transfer. ${ }^{20}$ Moreover, 4-NPOH is a nonfluorescent photoacid exhibiting fast vibrational cooling of the photoproduct, thereby leading to efficient formation of the aryolxide anion, 4-NPO-. In the case of a single species present, the ground state 4-NPOH species will be regenerated. On the other hand, in the presence of other species, new reactions in non-equilibrium conditions can be investigated. Herein, the last step involves reaction between aryloxide and water or acetic acid, which can be followed using time-gated UV-vis spectroscopy (laser flash photolysis) with high time resolution.

We have used the spherically-symmetric diffusion model ( $S S D P$ ) commonly employed to study excited state proton transfer. ${ }^{21,22}$ The Equation 1 presented below and all parameters employed in the present work are given in the SI. Additionally, the Debye-Smoluchowski model of reaction rates in 
solution gives the relation between rate constants $\left(k_{o b s}, k_{2}\right)$ and diffusion coefficient $\left(D_{A B}\right)$ of the reacting species:

$$
1 / k_{\text {obs }}=1 / 4 \pi \sigma D_{A B}+1 / k_{2}
$$

\section{2 | EXPERIMENTAL PROCEDURES}

All chemicals were purchased from Sigma-Aldrich. 4-nitrophenol was dissolved in water $([4-\mathrm{NPOH}]=$ $\left.1.0 \times 10^{-5} \mathrm{M}\right)$. Acetic acid was used in sodium acetate hydrate form $\left(\mathrm{CH}_{3} \mathrm{COONa}_{3} 3 \mathrm{H}_{2} \mathrm{O}\right)$ with addition of strong acid solution until values of $\mathrm{pH}$ above and below its $p K_{a}$ (4.76). The medium $\mathrm{pH}$ was varied from 4.0 to 5.2 and acetic acid concentration ranged from 0.5 to $10.0 \mathrm{mM}$. Ethylene glycol was mixed in solution until $60 \%$ with the purpose of increasing the medium shear viscosity, with or without ionic strength $([\mathrm{KCl}]=1.0 \mathrm{M})$. The viscosity measurements were performed using the following densimeter Anton Paar DMA 45000 with refractive index module RXA 170 and viscometer Anton Paarlov is 2000 M. Water was deionized, reaching resistance $>17 \mathrm{M} \Omega$.

\section{1 | Laser Flash Photolysis Measurements}

In this work, we used an Applied Photophysics, LKS80 Laser Flash Photolysis model for nanosecond transient absorption acquisitions on timescales up to microseconds with $3 \mathrm{~ns}$ time resolution. The pump source is the fourth harmonic of a Nd:YAG laser giving about $50 \mathrm{~mJ}$ at a wavelength of $266 \mathrm{~nm}$. We estimate that self-focusing, self-diffraction, and multi-photon absorption contribute negligibly to the observed signals. The sample compartment was positioned for focusing the excitation laser pump at 90 degrees geometry with respect to the probe. The probe light source was produced by Q-switching a Xenon Lamp with $150 \mathrm{~W}$ per pulse. Transient absorption signals were detected by a five-stage photomultiplier R928 operating from $190 \mathrm{~nm}$ to $920 \mathrm{~nm}$ and rise time of $3 \mathrm{~ns}$, placed after the monochromator in Czerny-Turner optical configuration with slits of 0.1 to $8.0 \mathrm{~mm}$ spacing. All measurements were performed in a fluorescence cuvette with an optical path length of $1.0 \mathrm{~cm}$. 
Measurement results were observed on the oscilloscope, Tektronix model DPO7000, and analyzed with the LKS Pro-date software.

\section{3 | RESULTS AND DISCUSSION}

\section{1 | Light-induced ground state system preparation: design considerations}

Using the photoacid cycle (see below) to guide our thinking and help choosing a good model chemical system for studying non-equilibrium proton transfer, we discuss each elementary step with a focus on how to improve the overall efficiency of ground state anion $\mathrm{A}^{-}$photo-production starting from the neutral ground state photo acid, AH. ${ }^{23-26}$ Specifically, we consider how to generate $\mathrm{A}^{-}$as quickly and efficiently as possible, and thus seek to minimize the time needed for each elementary photophysical and photochemical step involved: electronic excitation $\left(\mathrm{AH} \rightarrow \mathrm{AH}^{*}\right)$, excited state proton transfer $\left(\mathrm{AH}^{*} \rightarrow \mathrm{A}^{*^{-}}\right),{ }^{27}$ and non-radiative relaxation $\left(\mathrm{A}^{*-} \rightarrow \mathrm{A}^{-}\right) .{ }^{28}$ Once efficient ground state $\mathrm{A}^{-}$photoproduction has been achieved, it becomes possible to study chemical reactions that are otherwise difficult to follow, such as bimolecular proton transfer either back to chemical equilibrium or reacting with other species, as shown in Scheme1 in the case of 4-NPOH.

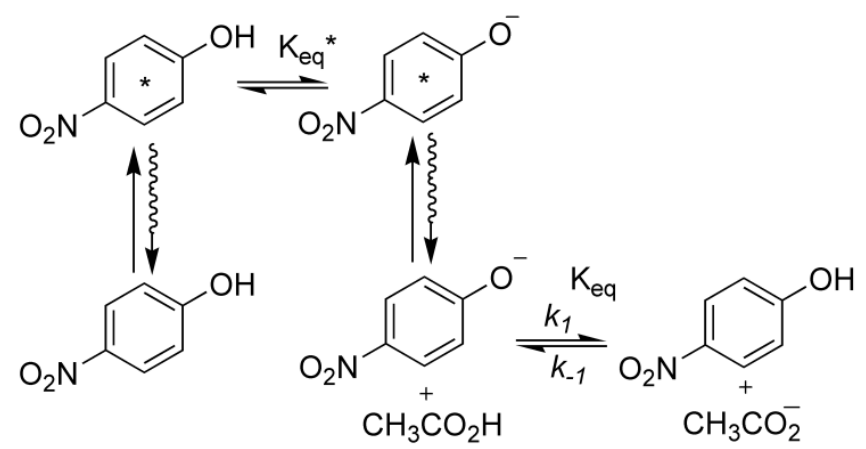

SCHEME 1 Transient formation of 4-NPO- in the ground state and subsequent bimolecular proton transfer reaction.

Since the pioneering work of Forster and Weller on photoacids, ${ }^{29,30}$ the elementary steps involved in excited state proton transfer $(E S P T)$ have been investigated with various frequency- and time-resolved spectroscopies as well as quantum chemistry and classical dynamics simulations. ${ }^{4}$ Regarding the first step in Scheme $1\left(\mathrm{AH} \rightarrow \mathrm{AH}^{*}\right)$, light absorption by $\mathrm{AH}$ leads to excited state $\mathrm{AH}^{*}$ formation in the 
ground state nuclear geometry. This step is limited by the period of an optical cycle, which is on the order of a few femtoseconds for visible light. ${ }^{31}$ In solution, this "frozen" configuration usually relaxes by structural rearrangement of nearby solvent molecules. Solvation dynamics is commonly characterized by two timescales: usually, the most pronounced component decays within hundreds of femtoseconds while the smaller diffusive component decays within a few picoseconds. ${ }^{32}$ Therefore, non-reactive solvation dynamics is essentially complete a few picoseconds after excitation. In the particular case of aromatic photoacids, light absorption and solvent relaxation have been studied for several systems. ${ }^{33}$ In such systems, light absorption is accompanied by intramolecular charge transfer (ICT) to either nearby or distal ring. In the case of photoacids containing a single aromatic ring, ICT leads to faster solvent rearrangement than for compounds with two rings. For example, in the case of 4cyanophenol, which is structurally similar to 4-nitrophenol, it has been shown that ICT stabilizes the anion much more than the acid, thus favoring the ESPT. ${ }^{34}$

Regarding the second step $\left(\mathrm{AH}^{*} \rightarrow \mathrm{A}^{*-}\right)$ in Scheme 1, excited state proton transfer timescales range from femtoseconds ${ }^{35}$ to seconds, ${ }^{36}$ depending on molecular structure. In the present work, we focus on the upper limit for $\mathrm{A}^{*^{-}}$formation rates. Proton transfer time constants ranging from one hundred femtoseconds up to a few picoseconds have been measured. ${ }^{36}$ The upper limit in ESPT reactions is associated with barrier-less excited state potential energy curves, whereas in bimolecular reactions, fast proton diffusion and proton transfer occur within solvent separated donor-acceptor complexes. Thus, solvation dynamics and proton transfer occur in similar timescales and are both important in optimizing ground state $\mathrm{A}^{-}$photo-production.

In the third step $\left(\mathrm{A}^{*^{-} \rightarrow} \rightarrow \mathrm{A}^{-}\right)$, usually $\mathrm{A}^{*^{-}}$decays to the ground state $\mathrm{A}^{-}$through a combination of radiative and radiationless processes. ${ }^{37}$ In order to efficiently produce $\mathrm{A}^{-}$, the electronic excitation energy of $\mathrm{A}^{*-}$ must be quickly converted to heat by internal conversion and vibrational cooling. Examples of fast vibrational energy relaxation include DNA bases ${ }^{38}$ and melanin pigments, ${ }^{39}$ where internal conversion occurs within $<500 \mathrm{fs}$, followed by vibrational cooling within a fewpicoseconds. ${ }^{40}$ 
Overall, we thus note that fast and efficient photo-production of $\mathrm{A}^{-}$involves several processes (solvation dynamics, proton transfer, internal conversion and vibrational cooling) occurring in similar timescales. As a result, we believe it is possible to generate $\mathrm{A}^{-}$from $\mathrm{AH}$ within approximately a picosecond, with an adequate choice of photoacids.

\section{2 | Numerical results.}

We employ a diffusion model of ground and excited state proton transfer based on the DebyeSmoluchowski spherically-symmetric diffusion equation (SSDP, see further details in the SI). ${ }^{21}$ Starting from the excited-state neutral photoacid, $\mathrm{AH}^{*}$, our goal is to evaluate the ground-state anion $\mathrm{A}^{-}$survival probability. To this end, we sought to find boundary conditions for this problem by numerically solving the SSDP for a range of parameters, as show in Figure 1 (see SI for parameters employed).

First, numerical solutions of the SSDP problem were used to calculate the survival probability of the ground state anion, $\mathrm{A}^{-}$, as a function of time. Except for the initial photo-excitation step which occurs in a few femtoseconds, we sought to investigate rate constants of each step in the overall process $\left(\mathrm{AH} \rightarrow \mathrm{AH}^{*} \rightarrow \mathrm{A}^{*-} \rightarrow \mathrm{A}^{-}\right)$. Given our initial goal of characterizing the ground state reaction of $\mathrm{A}^{-}$with proton, the optimum parameters should give survival probabilities matching the expected rate constant for the $\mathrm{A}^{-} \rightarrow \mathrm{AH}$ process. Figure 1 (left) shows the survival probabilities of each species in the photocycle, calculated using the SSDP model: $\mathrm{AH}^{*}$ (black), $\mathrm{A}^{*^{-}}$(blue), $\mathrm{A}^{-}$(green) and $\mathrm{AH}$ (red).
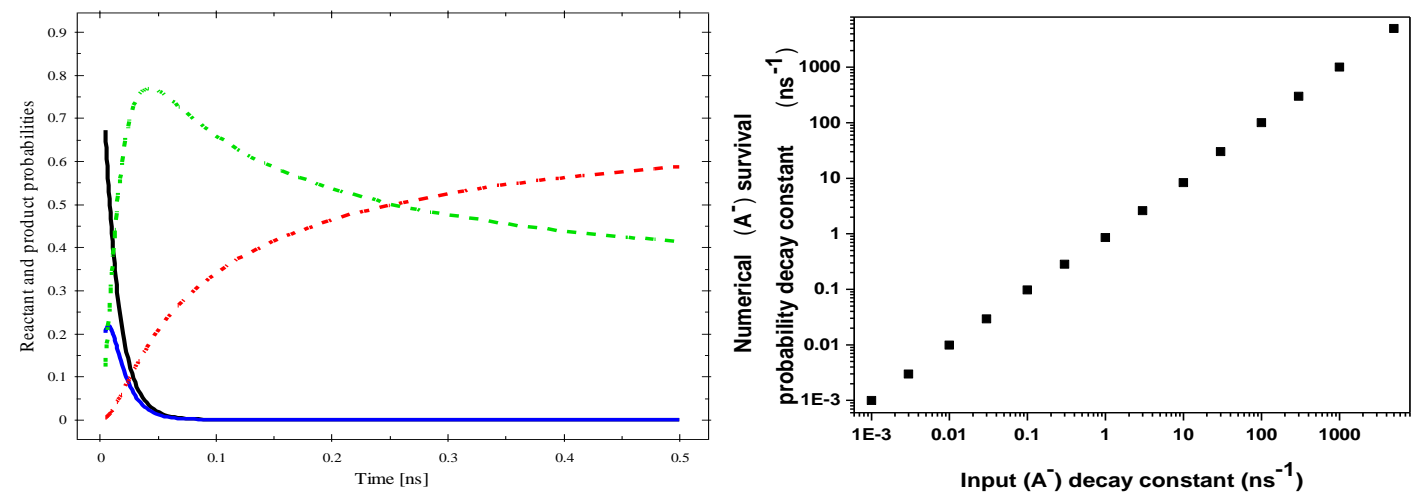
FIGURE 1 (left) Reactant and product survival probabilities for each species in the photoacid cycle: $\mathrm{AH}$ (black), AH*(blue), $\mathrm{A}^{*-}$ (green) and $\mathrm{A}^{-}$(red). (right) Survival probabilities of ground state anion $\left(\mathrm{A}^{-}\right)$for each expected $\mathrm{A}^{-}$decay constants, as obtained by numerical solution of the SSDP model.

With the parameters employed, the neutral excited state photoacid is quickly converted to excited state base. The ground state anion is generated within a short interval of time after excitation whereas the neutral acid AH is recovered on longer timescales. Depending on the choice of parameters, photoinduced production of the ground-state anion, which is the main purpose of the present study, can occur on a range of timescales. Figure 1 (right) shows a plot of numerically calculated survival probability decay constants (in units of $\mathrm{ns}^{-1}$ ) for each expected $\mathrm{A}^{-}$decay constant (in units of $\mathrm{ns}^{-1}$ ). The agreement between expected and numerical values is very good for time constants ranging from milliseconds down to one picosecond. This is characteristic of diffusion-controlled reactions investigated with the DebyeSmoluchowski approach, thus confirming that photo-production of $\mathrm{A}^{-}$in the ground-state can be experimentally realized even within a picosecond after photo-excitation. Such fast and efficient $\mathrm{A}^{-}$ production then allows the experimental study of bimolecular ground-state thermal reactions with high time resolution, leading to the formation of the neutral species AH on longer timescales.

Figure 2 shows numerically calculated survival probabilities of $\mathrm{A}^{-}$as a function of time for different values of the excited state sink function parameter, chosen to give decay time constants for the ground state protonation reaction $\left(\mathrm{A}^{-} \rightarrow \mathrm{AH}\right)$ matching our experimental results (see below). In the case of the photoacid cycle studied numerically, as long as photo-production of $\mathrm{A}^{-}$is sufficiently fast, it is indeed possible to follow the concentration of $\mathrm{A}^{-}$as a function of time as chemical equilibrium is restored $\left(\mathrm{A}^{-}\right.$ $\rightarrow \mathrm{AH})$, as shown in Figure 2 . 


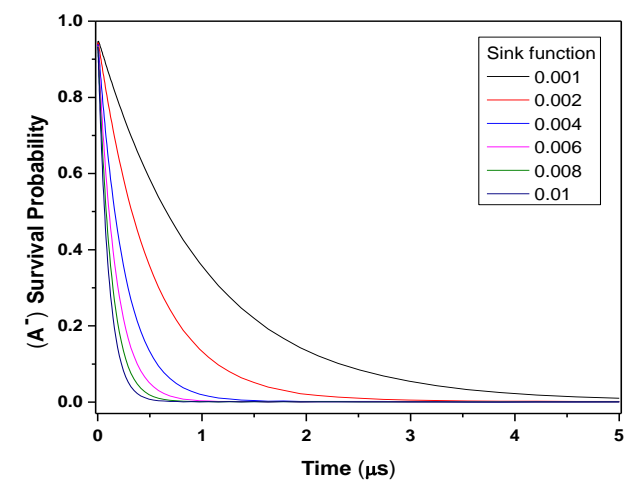

FIGURE 2 Survival probability of ground state anion $\left(\mathrm{A}^{-}\right)$as a function of time. The values of the sink function are chosen to match the experimentally obtained results.

\section{3 | Experimental study of Proton Transfer by Protolysis Mechanism}

Employing the laser flash photolysis technique, a short laser pulse is applied to acidic solution containing 4-nitrophenol, initiating the photo cycle. Pulsed white light is then used to probe the resulting kinetics leading to the appearance of the transient species 4-nitrophenolate, which in the present work may react with water or acetic acid to return to the neutral form. Previous works using photoacids characterize the transient species formed as either radical ${ }^{41}$ or cation radical. ${ }^{42}$ Although a similar technique was used, the medium in which the photoacid was submitted is different from that used in our work. The main focus of these works was on studying excited state hydrogen transfer $(E S H T)^{41}$ while the present study focuses on GSPT.

Figure 3 (top) shows our time resolved transient absorption spectra following 4-NPOH excitation at $266 \mathrm{~nm}$ and measured between in the 300 to $500 \mathrm{~nm}$ wavelength range. The main features of our experimental results are illustrated in Figure 3 (bottom): (i) fast decay (9 ns) of the ground state bleach signal near $300 \mathrm{~nm}$, associated with immediate disappearance of the neutral 4-NPOH species, and (ii) fast and efficient formation of $4-\mathrm{NPO}^{-}$in the ground state at $400 \mathrm{~nm}(29 \mathrm{~ns})$. These graphs are consistent with fast formation of the 4-NPO${ }^{-}$species in the ground state, as expected from our simulation results based on the SSDP model (Figure 2). Subsequently, equilibrium is attained and 4-NPO- is protonated, with a decrease in absorbance at $400 \mathrm{~nm}$, consistent with formation of 4-NPOH and concomitant increase in absorbance at $300 \mathrm{~nm}$. 

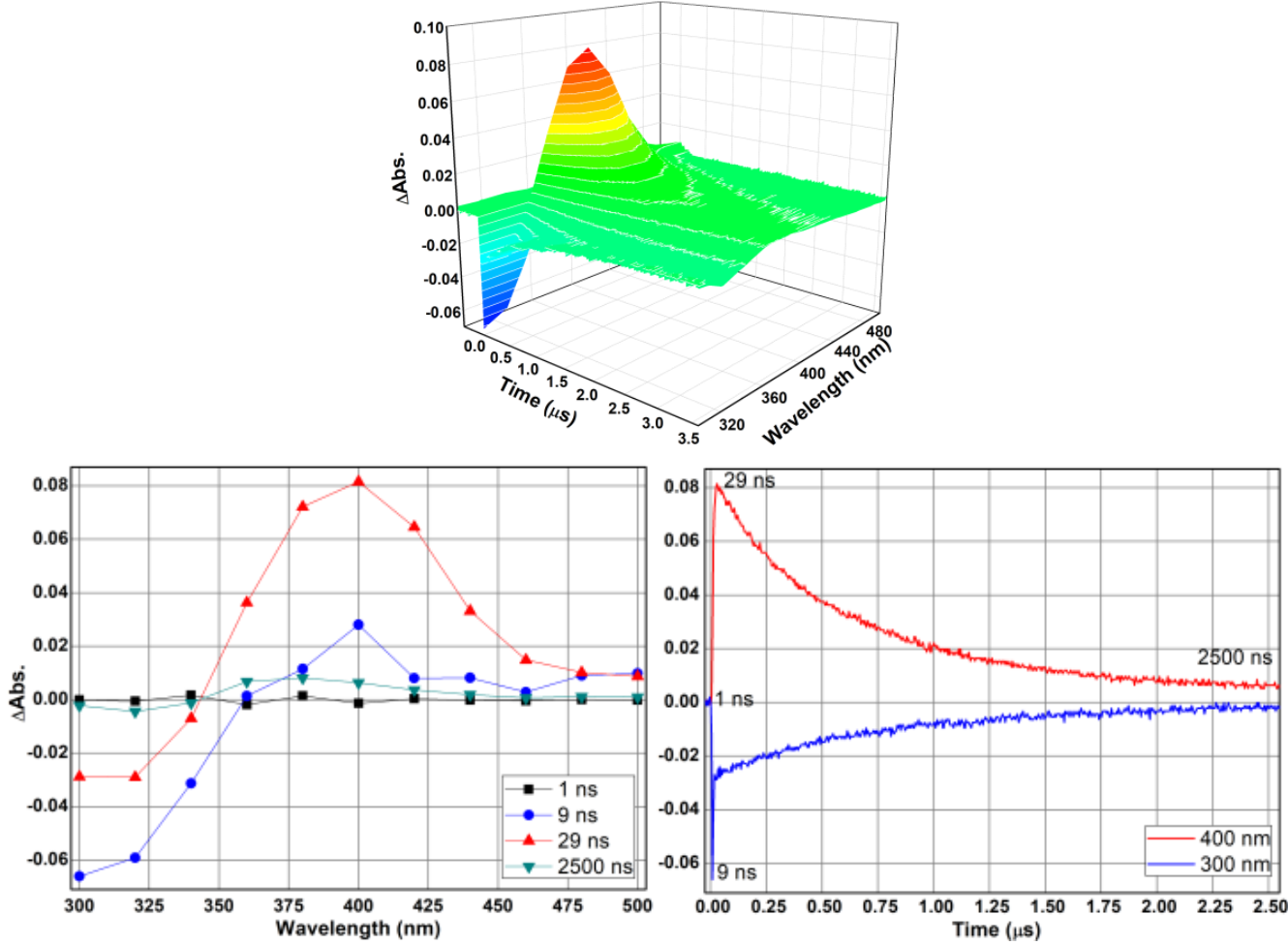

FIGURE 3 (top) Time-resolved transient absorption spectra following 4-NPOH excitation at $25{ }^{\circ} \mathrm{C}$ and $\mathrm{pH} 4.6$. (bottom, right) Transient absorption signals as a function of time at probe wavelength of $300 \mathrm{~nm}$ (blue) and $400 \mathrm{~nm}$ (red). (bottom, left) Transient absorption spectra at $1 \mathrm{~ns}$ (black), $9 \mathrm{~ns}$ (blue), $29 \mathrm{~ns}$ (red), and $2500 \mathrm{~ns}$ (green).

Figure 4 shows $\mathrm{pH}$-dependent kinetics of $4-\mathrm{NPO}^{-}$protonation in the $\mathrm{pH}$ range from 4.0 to 5.2 , with faster decay under more acidic conditions. Each plot corresponds to a single acetic acid concentration, ranging from 0.5 to $10 \mathrm{mM}$ such that, for each $\mathrm{pH}$, faster decays are observed with increasing acetic acid concentration. In these graphs it is possible to see the similarity with the result presented by Figure 2. Therefore, changes in $\mathrm{pH}$ or concentration of $\mathrm{AcOH}$ can be compared to the simulations based on the SSDP model.
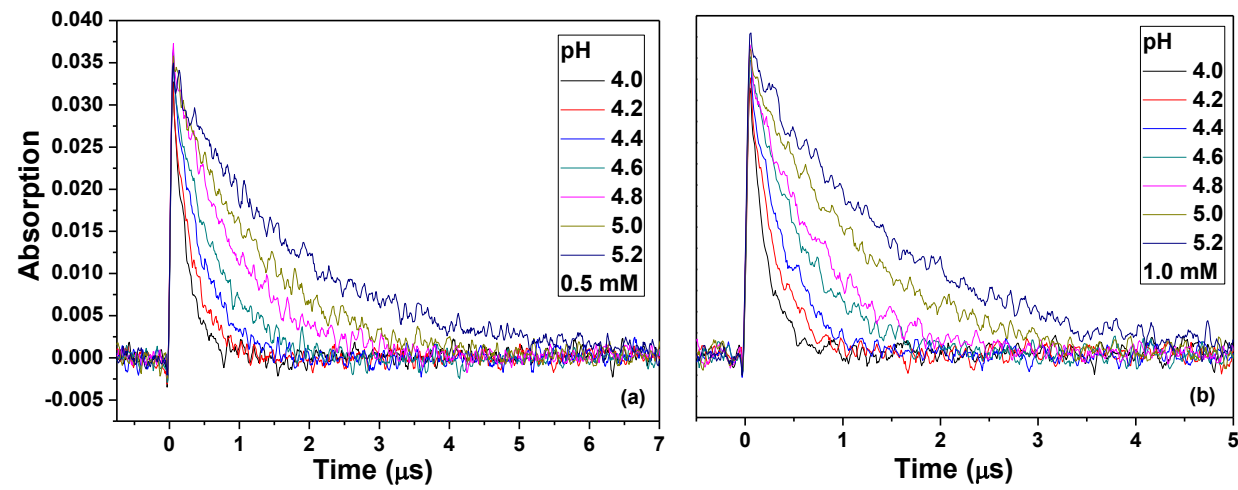

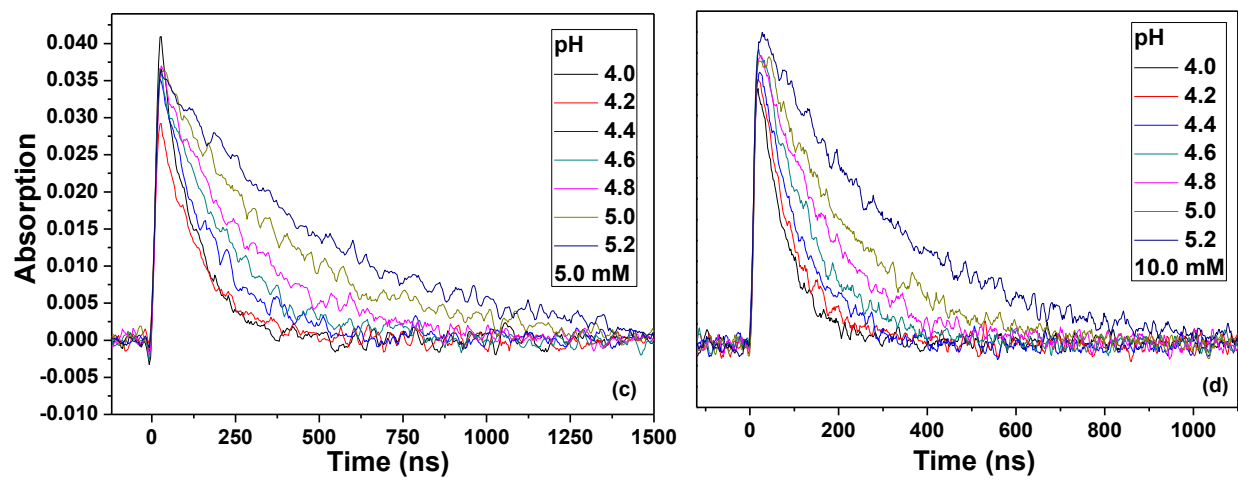

FIGURE 4 Decay of 4-NPO- in $[\mathrm{AcOH}]$ (a) 0.5 , (b) 1.0 , (c) 5.0 and (d) $10.0 \mathrm{mM}, \mathrm{pH} 4.0-5.2$ at $400 \mathrm{~nm}$.

Non-linear least squares fitting of the data shown in Figure 4 gives the observed rate constants in Figure 5. Lower $\mathrm{pH}$ (4.0) and higher concentration values $\mathrm{AcOH}(10.0 \mathrm{mM})$ showed the highest observed rate constant values, with a linear increase in rate constant at all $\mathrm{pH}$ values examined. The intercept of the lines shown in Figure 5 (corresponding to $[\mathrm{AcOH}]=0$ ) were used to characterize rate constants as a function of $\mathrm{H}_{3} \mathrm{O}^{+}$.

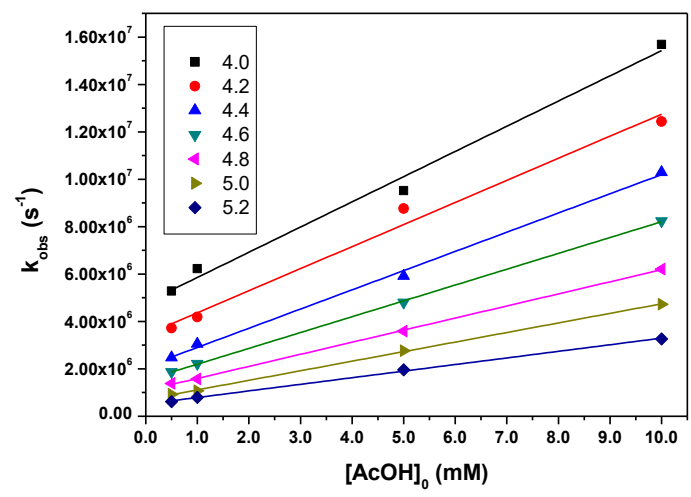

FIGURE 5 Observed rate constants with increase of concentration $\mathrm{AcOH}$ and $\mathrm{pH}$ variation for 4-NPO-

The results shown above are consistent with Eigen's ${ }^{43}$ treatment, since 4-nitrophenolate protonation reactions by water molecules occur with a fast and direct second order rate constant (about $3.5 \times 10^{10}$ L. $\left.\mathrm{mol}^{-1} \cdot \mathrm{s}^{-1}\right) .{ }^{44}$ On other hand, when the medium is made slightly acidic by adding a weak acid such as acetic acid, the reaction will also occur by protolysis mechanism. In this case, proton transfer from hydrogen donor to nitrophenolate can be accomplished by either carboxylic acid or by hydronium ion. 
Equation 2 accounts for the $\mathrm{pH}$ dependent molar fraction of acetic acid and acetate species present in solution:

$$
k_{\text {obs }}=\left(k_{A c O H}^{P} \chi_{A c O H}+k_{A c O}^{D} \chi_{A c O}\right)[A c O H]_{0}+k_{H}^{P}\left[H_{3} O^{+}\right]+k_{H}^{D}
$$

The linear relationship is consistent with Equation 2 and the linear coefficient corresponds to the deprotonation constant $\left(k_{\mathrm{H}}{ }^{\mathrm{D}}\right)$ of 4-NPOH, while the slope corresponds to the rate constant for the protonation $\left(k_{\mathrm{H}}{ }^{\mathrm{P}}\right)$ of $4-\mathrm{NPO}^{-}$with hydronium ion (in Figure 6 (left) and values given in Table 1).

Figure 6 (right) shows the second order rate constants calculated from the slope of the plots in Figure 5 as a function of the molar fraction of $\mathrm{AcOH}$. The linear relationship allows us to calculate the deprotonation rate constant $\left(k_{\mathrm{AcO}}{ }^{\mathrm{D}}\right)$ and the protonation constant $\left(k_{\mathrm{AcOH}}{ }^{\mathrm{P}}\right)$ for the reaction with acetic acid and the calculated values of constants.

The values fit the observed rate constant (black dots in Figure 7) as a function of $\mathrm{pH}$ and acetic acid concentration. The calculated values correspond to the continuous red line, which was calculated using the rate constants given in Table 1 . The agreement shows the validity of Eigen's treatment and the quality of the experimental data obtained with the new approach. We note that this study was performed in slightly acidic medium ( $\mathrm{pH}$ between 4.0 and 5.2) such that the reaction occurs concurrently by protolysis. ${ }^{43,44}$
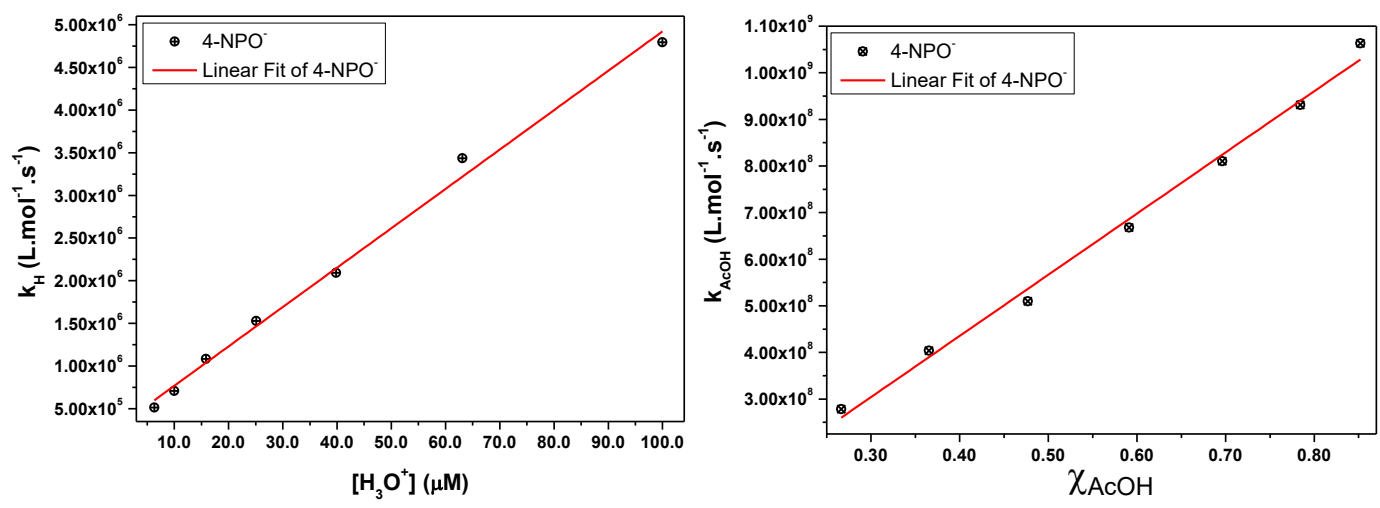

FIGURE 6 (left) Rate constant for the reaction of 4-NPO- with hydronium ion as a function of the $\left[\mathrm{H}_{3} \mathrm{O}^{+}\right]$. (right) Rate constant with increase molar fraction of $\mathrm{AcOH}$ for 4-NPO'. 


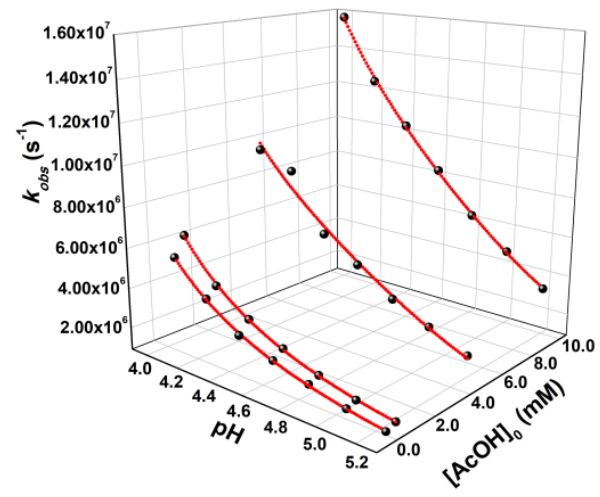

FIGURE 7 Non-linear curve fit (red line) of $k_{o b s}$ as a function of $\mathrm{pH}$ and [AcOH] for 4-NPO- The black dots are observed values of $k_{\text {obs }}$.

The constants with respect to $\mathrm{H}_{3} \mathrm{O}^{+}$are greater than the constants with respect to $\mathrm{AcOH}$. Therefore, $K_{a}=$ $k^{\mathrm{D} / k^{\mathrm{P}}}$ (from the last two columns in Table 1) we find that the $p K_{a}$ is 5.17 . The rate constants derived from the relaxation back to equilibrium should reproduce the value of the $p K_{a}$ of $\mathrm{AcOH}$ (4.76). The discrepancy between the calculated value and the literature value is related to the aforementioned competition between distinct mechanisms in this $\mathrm{pH}$ range (by $\mathrm{AcOH}$ and $\mathrm{H}_{3} \mathrm{O}^{+}$protonation). Previous work have shown that salts (e.g., fluoride and acetate) of weak acids with $p K_{a}$ values between ground and excited state acidity constants react with the photoacid in the excited state. ${ }^{45}$

TABLE 1 Protonation and deprotonation rates constant for the reactions of 4-NPO//4-NPOH and acetic acid buffer media.

\begin{tabular}{|c|c|c|c|c|c|}
\hline & $p K_{a}$ & $k_{\mathrm{AcOH}}{ }^{\mathrm{P}}\left(\mathbf{L} \cdot \mathrm{mol}^{-1} \cdot \mathrm{s}^{-1}\right)$ & $k_{\mathrm{AcO}}{ }^{\mathrm{D}}\left(\mathrm{L} \cdot \mathrm{mol}^{-1} \cdot \mathrm{s}^{-1}\right)$ & $k_{\mathrm{H}} \mathbf{P}\left(\mathbf{L} \cdot \mathrm{mol}^{-1} \cdot \mathrm{s}^{-1}\right)$ & $k_{\mathrm{H}^{-}} \mathbf{D}\left(\mathrm{s}^{-1}\right)$ \\
\hline & 7.15 & $1.31 \pm 0.05 \times 10^{9}$ & $8.96 \pm 2.81 \times 10^{7}$ & $4.61 \pm 0.15 \times 10^{10}$ & $3.08 \pm 0.75 \times 10^{5}$ \\
\hline
\end{tabular}

As expected, the rate constant for proton transfer between $\mathrm{AcOH}$ and 4-NPO- increases with temperature and the data shows the expected dependence in terms of both Arrhenius (Fig. S1 in the SI) and Eyring treatments (Fig. S2 in the SI) and the calculated activation parameters are given in Table 2.

TABLE 2 Arrhenius parameters and activation parameters of 4-NPO'. 


\begin{tabular}{|c|c|c|c|c|}
\hline$E_{a}\left(\mathrm{kcal} . \mathrm{mol}^{-1}\right)$ & $\log A$ & $\Delta \mathbf{H}^{\ddagger}\left(\right.$ kcal.mol $\left.^{-1}\right)$ & $\Delta \mathrm{S}^{\ddagger}\left(\mathrm{cal} \mathrm{mol} \mathbf{l}^{-1} \cdot \mathbf{K}^{-1}\right)$ & $\Delta G^{\ddagger}\left(\right.$ kcal.mol $\left.^{-1}\right)$ \\
\hline 2.61 & 8.49 & 2.01 & -21.70 & 8.48 \\
\hline
\end{tabular}

The activation energy $E_{a}$ is small $\left(2.61 \mathrm{kcal}^{\mathrm{mol}}{ }^{-1}\right)$ and the pre-exponential parameter $A(\log A=8.49)$ are small, a fact fully reasonable since proton transfer in solution happens quickly, especially for reactions between a negatively charged species (4-nitrophenolate) receiving a proton from an acidic proton donor $\mathrm{AH}$ (acetic acid). The activation enthalpy $\left(\Delta \mathrm{H}^{+}=2.01 \mathrm{kcal}^{+} \mathrm{mol}^{-1}\right)$ and large and negative activation entropy $\left(\Delta \mathrm{S}^{\ddagger}=-21.7 \mathrm{cal} . \mathrm{mol}^{-1} \mathrm{~K}^{-1}\right)$ are consistent with a bimolecular reaction, and slowing down the reaction as shown by the fact that the Gibbs activation free energy is larger than the activation enthalpy. This set of parameters presents a small barrier of activation energy that is already expected and observed in other works. ${ }^{13}$

All the evidence presented here (rate constants and energy values) are consistent with the Eigen proton transfer by protolysis mechanism involving the 4-nitrophenolate anion. ${ }^{43,44}$ The start of the photo cycle with 4-NPOH is in accordance with the SSDP model and the transient species formed at $400 \mathrm{~nm}$ is characterized by presence of 4-NPO'.

\section{4 | Viscosity Effect on Proton Transfer by Debye-Smoluchowski Diffusion Model}

The connection between the numerical results obtained from the SSDP model and experimental results on the protolysis mechanism for ground state proton transfer was further studied. The numerical model used the Debye-Smoluchowski equation to calculate the possibilities of survival of an anion $\mathrm{A}^{-}$. The same equation can be applied in the study with the aid of laser flash photolysis. However, one parameter stands out among the others to control the diffusion in solution, namely, the shear viscosity.

In order to study the effect of viscosity on reaction rates, mixtures of water/AcOH and ethylene glycol were employed. Table 3 shows the effect of volume percent solution (v/v) on viscosity, relative diffusion coefficient and ionic strength (IS, with $1.0 \mathrm{M} \mathrm{KCl}$ ) between species. It is observed that increasing ethylene glycol molar fraction increases the medium viscosity whereas the relative diffusion 
coefficient decreases. Figure 8 (left) shows the relaxation time for the system and the shear viscosity variation. Figure 8 (right), shows the positive correlation between the inverse of observed rate constant for the system and the inverse of the relative diffusion coefficient $\left(1 / D_{A B}\right)$, both as a function of ethylene glycol concentration in solution - in this case, the conditions are $[\mathrm{KCl}]=1.0 \mathrm{M},\left[\mathrm{AcOH} / \mathrm{AcO}^{-}\right]=10.0$ $\mathrm{mM}$ and $\mathrm{pH}$ 4.2. The linear fit is done by the following Equation 1 derived from the DebyeSmoluchowski theory.

TABLE 3 Shear viscosity, diffusion coefficients and observed rate constant with increase of ethylene glycol in the medium in absence and presence of ionic strength (IS, $[\mathrm{KCl}]=1.0 \mathrm{M}$ ).

\begin{tabular}{ccccccc}
\hline $\mathbf{C}_{2} \mathbf{H}_{4}(\mathbf{O H})_{2}(\mathbf{V} / \mathbf{V})$ & $\eta_{S}(\mathbf{m P a} . \mathbf{S})$ & $\eta \boldsymbol{s}^{(\mathbf{I S})}(\mathbf{m P a} . \mathbf{S})$ & $\boldsymbol{D}_{\boldsymbol{A B}}\left(\mathbf{m}^{2} \cdot \mathbf{s}^{-1}\right)$ & $\boldsymbol{D}_{\boldsymbol{A B}}{ }^{(\mathbf{I S})}\left(\mathbf{m}^{2} \cdot \mathbf{s}^{-1}\right)$ & $\boldsymbol{k}_{\text {obs }}\left(\mathbf{s}^{-1}\right)$ & $\boldsymbol{k}_{\text {obs }}{ }^{(\mathbf{I S})}\left(\mathbf{s}^{-1}\right)$ \\
\hline 0 & 1.12 & 1.05 & $1.76 \times 10^{-9}$ & $1.87 \times 10^{-9}$ & $1.48 \times 10^{7}$ & $1.45 \times 10^{7}$ \\
$10 \%$ & 1.33 & 1.27 & $1.48 \times 10^{-9}$ & $1.54 \times 10^{-9}$ & $1.27 \times 10^{7}$ & $1.33 \times 10^{7}$ \\
$20 \%$ & 1.70 & 1.77 & $1.15 \times 10^{-9}$ & $1.14 \times 10^{-9}$ & $1.13 \times 10^{7}$ & $1.15 \times 10^{7}$ \\
$30 \%$ & 2.26 & 2.25 & $8.70 \times 10^{-10}$ & $8.74 \times 10^{-10}$ & $1.00 \times 10^{7}$ & $1.01 \times 10^{7}$ \\
$40 \%$ & 2.93 & 3.05 & $6.72 \times 10^{-10}$ & $6.44 \times 10^{-10}$ & $8.53 \times 10^{6}$ & $8.08 \times 10^{6}$ \\
$50 \%$ & 3.84 & 3.99 & $5.12 \times 10^{-10}$ & $4.93 \times 10^{-10}$ & $7.21 \times 10^{6}$ & $6.90 \times 10^{6}$ \\
$60 \%$ & 4.94 & 5.48 & $3.98 \times 10^{-10}$ & $3.59 \times 10^{-10}$ & $6.49 \times 10^{6}$ & $6.05 \times 10^{6}$ \\
\hline
\end{tabular}
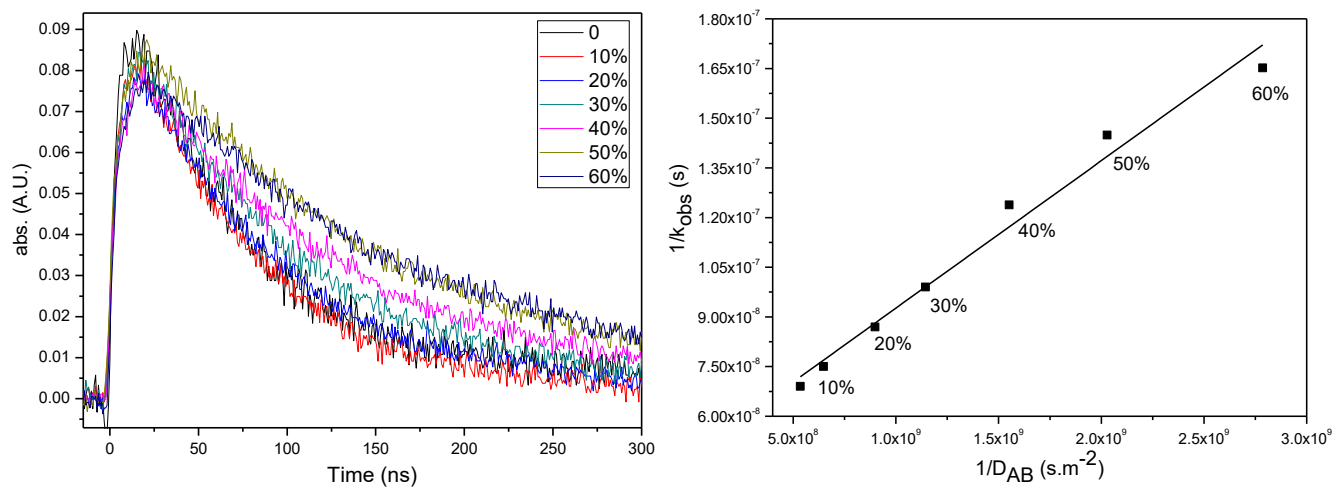

FIGURE 8 (left) Relaxation time in nanoseconds under influence of ethylene glycol molar fraction. (right) Observed rate constants and diffusion coefficients to $4-\mathrm{NPO}^{-}$with linear curve fit. The conditions are ionic strength $[\mathrm{KCl}]=1.0 \mathrm{M}$, buffer $\left[\mathrm{AcOH} / \mathrm{AcO}^{-}\right]=10.0 \mathrm{mM}$ and $\mathrm{pH} 4.2$.

Regarding the values obtained for the observed rate constants, the viscosity effect is more pronounced in relation to the effect of ionic strength. We can also highlight here, studies already mentioned in which 
the increase in the viscosity of the medium, with glycerol, similarly affected the diffusion and the addition of electrolyte minimally modified the diffusion control. ${ }^{6,7}$

Table 4 shows the steady value of the second order found from the linear coefficient obtained in the graph of Figure 8. In addition other second order constant the ratio to 4-NPOH was maintained, with or without ionic strength.

TABLE 4 Second order rate constants of proton transfer to 4-NPO- by adjust in non-linear curve fit and by DebyeSmoluchovski model with or without ionic strength (IS).

\begin{tabular}{|c|c|c|c|c|c|}
\hline \multirow{2}{*}{ 4-NPO- } & $p K_{a}$ & $k_{\mathrm{AcOH}}{ }^{\mathrm{P}}\left(\mathbf{L} \cdot \mathrm{mol}^{-1} \cdot \mathrm{s}^{-1}\right)$ & $k_{\mathrm{H}} \mathbf{P}\left(\mathbf{L} \cdot \mathrm{mol}^{-1} \cdot \mathrm{s}^{-1}\right)$ & $k_{2}\left(\mathbf{L} \cdot \mathrm{mol}^{-1} \cdot \mathrm{s}^{-1}\right)$ & $k_{2}{ }^{(\mathrm{IS})}\left(\mathbf{L} \cdot \mathrm{mol}^{-1} \cdot \mathrm{s}^{-1}\right)$ \\
\hline & 7.15 & $1.31 \times 10^{9}$ & $4.61 \times 10^{10}$ & $2.07 \times 10^{9}$ & $2.08 \times 10^{9}$ \\
\hline
\end{tabular}

In this case, the addition of ethylene glycol is treated as if it only affected the viscosity of the medium (from pure water and $\eta_{s}=1.05 \mathrm{mPa} . \mathrm{S}$ to $60 \% \mathrm{v} / \mathrm{v}$ ethylene glycol and $\eta_{s}=5.48 \mathrm{mPa} . \mathrm{S}$ ). The variation in observed rate constants with increasing ethylene glycol \% v/v was measured at buffer concentration and $\mathrm{pH}$, such that the amount of transferable protons remained constant also. Thus, the analysis focused mainly on the diffusion of species and with less influence from the solvation network formed in solution. The negligible changes in the observed rate constants in relationship to ionic strength also are consistent with diffusion mechanism.

\section{$4 \mid$ CONCLUSION}

In conclusion, we have presented numerical and experimental results on the ground state proton transfer in solution. Spherically Symmetric Diffusion model was initially employed to calculate survival probabilities of each species in the proton transfer photocycle, and then we investigated how to use the proton transfer photocycle to produce the ground state anion efficiently. The results are generally valid within the framework of the spherically symmetric diffusion model of chemical reactions for arbitrary timescales ranging from picoseconds to hours. Using 4-nitrophenol (4-NPOH) as our model photoacid system, we have measured time-resolved transient absorption spectra following 4-NPOH excitation as a 
function of $\mathrm{pH}, \mathrm{AcOH}$ concentration, temperature, and viscosity. The time-resolved spectroscopy results were employed to study the protonation and deprotonation for the reactions of 4-nitrophenolate and acetic acid. Overall, the combined numerical and experimental work allowed us to characterize the ground electronic state reaction involving 4-nitrophenolate and acetic acid even at $\mathrm{pH}$ values well below the $p K_{a}$ of $4-\mathrm{NPOH}$.

\section{ACKNOWLEDGEMENTS}

The authors acknowledge to Educational development group (CAPES), Scientific development group (CNPq) and Brazilian funding INCT, FAPESP and FAPESC.

\section{SUPPORTING INFORMATION}

Additional supporting information may be found online in the Supporting Information section at the end of the article.

\section{REFERENCES}

[1] C. F. Bernasconi, in Relaxation kinetics, Academic Press, 1976, 288.

[2] A. H. Zewail, J. Phys. Chem. A 2000, 104, 5660.

[3] S. Mukamel, in Principles of Nonlinear Optical Spectroscopy, Oxford University Press, 1995, 79.

[4] A. P. Gamiz-Hernandez, A. Magomedov, G. Hummer, V. R. I. Kaila, J. Phys. Chem. B 2015, 119, 2611.

[5] M. Vester, T. Staut, J. Enderlein, G. Jung, J. Phys. Chem. Lett. 2015, 6, 1149.

[6] B. Cohen, D. Huppert, N. Agmon, J. Am. Chem. Soc. 2000, 122, 9838.

[7] B. Cohen, D. Huppert, N. Agmon, J. Phys. Chem.A 2001, 105, 7165.

[8] M. Rapoport, C. K. Hancock, E. A. Meyers, J. Am. Chem. Soc. 1961, 83, 3489.

[9] C. K. Hancock, A. P. Clague, J. Am. Chem. Soc. 1964, 86, 4942.

[10] E. L. Wehry, L. B. Rogers, J. Am. Chem. Soc. 1965, 87, 4234.

[11] M. Krauss, J. O. Jensen, H. F. Hameka, J. Phys. Chem. 1994, 98, 9955.

[12] G. Schuurmann, R. K. Somashekar, U. Kristen, Environ. Toxicol. Chem. 1996, 15, 1702. 
[13] S. Kanego, S. Yotoriyama, H. Koda, S. Tobita, J. Phys. Chem. A 2009, 113, 3021.

[14] M. Miyazaki, A. Kawanishi, I. Nielsen, I. Alata, S. Ishiuchi, C. Dedonder, C. Jouvet, M. Fujii, J. Phys. Chem. A 2013, 117, 1522.

[15] S. K. Panja, N. Dwivedi, S. Saha, Phys. Chem. Chem. Phys. 2016, 18, 21600.

[16] V. Kavitha, K. Palanivelu, J. Photoch. Photobio. A: Chem. 2005, 170, 83.

[17] L. Palmisano, M. Schiavello, A. Sclafani, C. Martin, I. Martin, V. Rives, Catal. Lett. 1994, 24, 303.

[18] D. W. Chen, A. K. Ray, Water Res. 1998, 32, 3223.

[19] D. D. Perrin, in Dissociation constants of organic bases in aqueous solution, Butterworths, 1965 , 473.

[20] J. R. Mora, A. J. Kirby, F. Nome, J. Org. Chem. 2012, 77, 7061.

[21] E. B. Krissinel', N. Agmon, J. Comp. Chem. 1996, 17, 1085.

[22] S. R. Logan, in Fundamentals of Chemical Kinetics, Longman House, Burnt Mill, Harlow, 1996, 264.

[23] T. Förster, S. Völker, Chem. Phys. Lett. 1975, 34, 1.

[24] K. K. Smith, K. J. Kaufmann, D. Huppert, M. Gutman, Chem. Phys. Lett. 1979, 64, 522.

[25] M. Gutman, D. Huppert, E. Pines, J. Am. Chem. Soc. 1981, 103, 3709.

[26] E. Pines, D. Huppert, J. Phys. Chem. 1983, 87, 4471.

[27] J. T. Hynes, J. P. Klinman, H. H. Limbach, R. L. Schowen, in Hydrogen-Transfer Reactions, Wiley-VCH, 2007, 377.

[28] N. J. Turro, in Modern molecular photochemistry of organic molecules, University Science Books, 2010, 13.

[29] T. Förster, Z. Elektrochem. 1950, 54, 531.

[30] A. Weller, Prog. React. Kinet. 1961, 1, 187.

[31] T. Brabec, F. Krausz, Rev. Mod. Phys. 2000, 72, 545.

[32] R. M. Stratt, M. Maroncelli, J. Phys. Chem. 1996, 100, 12981.

[33] N. Agmon, J. Phys. Chem. A 2005, 109, 13.

[34] G. Granucci, J. T. Hynes, P. Millie, T. H. Tran-Thi, J. Am. Chem. Soc. 2000, 122, 12243. 
[35] M. Rini, B. Z. Magnes, E. Pines, E. Nibbering, Science 2003, 301, 349.

[36] R. M. D. Nunes, M. Pineiro, L. G. Arnaut, J. Am. Chem. Soc. 2009, 131, 9456.

[37] J. R. Lakowicz, in Principles of Fluorescence Spectroscopy, Springer, 2006, 8.

[38] T. Gustavsson, A. Banyasz, E. Lazzarotto, D. Markovitsi, G. Scalmani, M. J. Frisch, V. Barone, R. Improta, J. Am. Chem. Soc. 2006, 128, 607.

[39] P. Meredith, J. Riesz, Photochem. Photobiol. 2004, 79, 211.

[40] C. T. Middleton, B. Cohen, B. Kohler, J. Phys. Chem. A 2007, 111, 10460.

[41] M. Miyazaki, R. Ohara, C. Dedonder, C. Jouvet, M. Fujii, Chem. Eur. J. 2018, 24, 881.

[42] S. Zhao, H. Ma, M. Wang, C. Cao, J. Xiong, Y. Xu, S. Yao, J. Hazard. Mater. 2010, $180,86$.

[43]M. Eigen, Angew. Chem. Int. Edit. 1964, 3, 1.

[44] M. Eigen, K. Kustin, J. Am. Chem. Soc. 1960, 82, 5952.

[45] A. L. Maçanita, P. F. A. Moreira Jr., J. C. Lima, F. H. Quina, C. Yihwa, C. Vautier-Giongo, J. Phys. Chem. A 2002, 106, 1248. 


\section{Entry for the Table of Contents}

Graphic for Table of Contents:

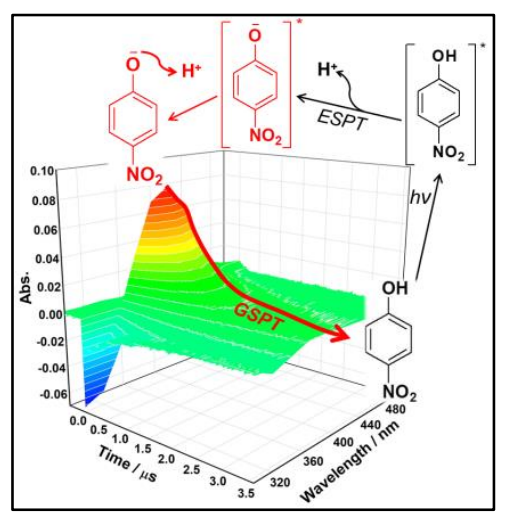

The Table of Contents text:

We performed laser flash photolysis measurements and numerical simulation of protonation rate constants of 4-nitrofenolate as a function of $\mathrm{AcOH}, \mathrm{pH}$, temperature and viscosity. Overall, the work describes a study of fast proton transfer reactions in non-equilibrium conditions in the ground state. 


\section{SUPPORTING INFORMATION}

\section{Time-resolved Study of Light-induced Ground State Proton Transfer from Acid}

\section{Medium to 4-nitrophenolate}

Leandro Scorsin | Letícia S. Martins | Haidi D. Fiedler | René A. Nome | Faruk Nome

\section{Spherically-Symmetric Diffusion Model}

The model considers the probability density, $p(r, t)$ for $\mathrm{AH}^{*}(\mathrm{r}) \rightarrow \mathrm{A}^{*}-+\mathrm{H}+$ at some time $t$ following photoexcitation. The $\mathrm{AH}^{*}$ signal is given by $P(t)$, whereas the $\mathrm{A}^{*-}$ signal is given by $S(t)$, the survival probability of the separated pair:

$$
\mathrm{S}(t) \equiv 4 \pi \int_{a}^{\infty} p(r, t) r^{2} d r
$$

Under the boundary conditions discussed above, the $\mathrm{A}^{-}$population is given by $1-P(t)-S(t) .{ }^{1}$ As a result, the kinetic equation for $p(r, t)$ is given by:

$$
\begin{gathered}
\frac{\partial}{\partial t} p(r, t)=\left[r^{-2} \frac{\partial}{\partial r} D r^{2} e^{V(r)} \frac{\partial}{\partial r} e^{V(r)}-k_{0}^{\prime}\right] p(r, t) \\
+\left[k_{d} \mathrm{P}(t)-\left(k_{a}+k_{q}\right) p(r, t)\right] \frac{\delta(r-a)}{4 \pi a^{2}} \\
\frac{\partial}{\partial t} p(r, t)=k_{a} p(a, t)-\left(k_{d}+k_{0}\right) \mathrm{P}(t)
\end{gathered}
$$

Where $D$ is the relative diffusion coefficient, $\mathrm{V}(\mathrm{r})$ is the attractive Coulomb potential between $\mathrm{A}^{-}$and $\mathrm{H}^{+}$, $k_{a}$ is the association rate constant, $k_{q}$ is the proton quenching rate constant, $k_{0}$ is the $\mathrm{AH}^{*}$ excited state decay constant, $k_{0}^{\prime}$ is the $\mathrm{A}^{*^{-}}$excited state decay constant, $k_{d}$ is the dissociation rate constant, and $a$ is the distance at which the reaction occurs.

All the numerical calculations were performed with the freely available SSDP software, version 2.66. ${ }^{1}$ Table $\mathrm{S} 1$ lists the parameters employed in the calculations shown in Figures 1 and 2.

TABLE S1 Sample parameters employed in the determination of ground- and excited-state survival and recombination probabilities employed SSDP software. 
Parameter

Excited state dissociation rate (angstrom/ns)

Excited state recombination rate (1/ns)

Initial distribution

Interaction potential

Inner radius for diffusion spaces (angstrom)

Diffusion coefficient $\left(\mathrm{cm}^{2} / \mathrm{s}\right)$

Decay function to ground state $(1 / \mathrm{ns})$

Ground state forward rate constant (angstrom/ns)

Ground state backward rate constant (1/ns)
Value

0.74

80

0

Coulomb

7

$9.3 \times 10^{-5}$

Tunable from 0.001 to 0.01 (see Figure 2 in Main

Text)

35

$1.0 \times 10^{-6}$

\section{The Diffusion Model of Debye-Smoluchowski}

The solution reactions theory explains the fact that reagents $\mathrm{A}$ and $\mathrm{B}$ could collide and react or do not colide, in dependence of diffusion in the reactional medium. The Stokes-Einstein equation express diffusion coeficient $D_{A B}$ in terms of the radius of the species A and $\mathrm{B}\left(r_{A B}\right)$ and the viscosity coefficient $\eta$ of the solvent. ${ }^{2}$

$$
D_{A B}=k_{B} T / 6 \pi r_{A B} \eta
$$

A different lower boundary condition has been proposed as an alternative to that used by DebyeSmoluchowski. The concentration of the diffusing species at radius $r_{A B}$ is equal distance from A to B $\sigma$ and in the steady state flux we consider one species (hypothetically $B$ ) with concentration $\left(c_{B}\right)$ maintained uniform. In this case, can be adopted a rate constant $k$ that would be applicable. ${ }^{2}$

$$
\mathrm{c}_{\mathrm{B}}=\mathrm{c}_{\infty}-k \mathrm{c}_{\mathrm{B}} / 4 \pi \sigma D_{A B}
$$


However, it is the operative second order rate constant $k_{2}$ which is experimentally accesible.

$$
k_{2}=k \mathrm{c}_{\mathrm{B}} / \mathrm{c}_{\infty}=4 \pi \sigma D_{A B} / 1+\left(\frac{4 \pi \sigma D_{A B}}{k}\right)
$$

Rearranging we have Equation 1 described in introduction:

$$
1 / k_{\text {obs }}=1 / 4 \pi \sigma D_{A B}+1 / k_{2}
$$

\section{Effect of Temperature on Proton Transfer}

Figure S1 shows the linear fit for the Arrhenius parameters for 4-NPO ${ }^{-}$and shows the value of activation energy $E_{a}$ and the pre-exponential parameter $A$.

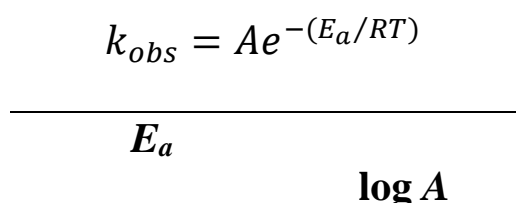

(kcal.mol $\left.{ }^{-1}\right)$

2.61

8.49

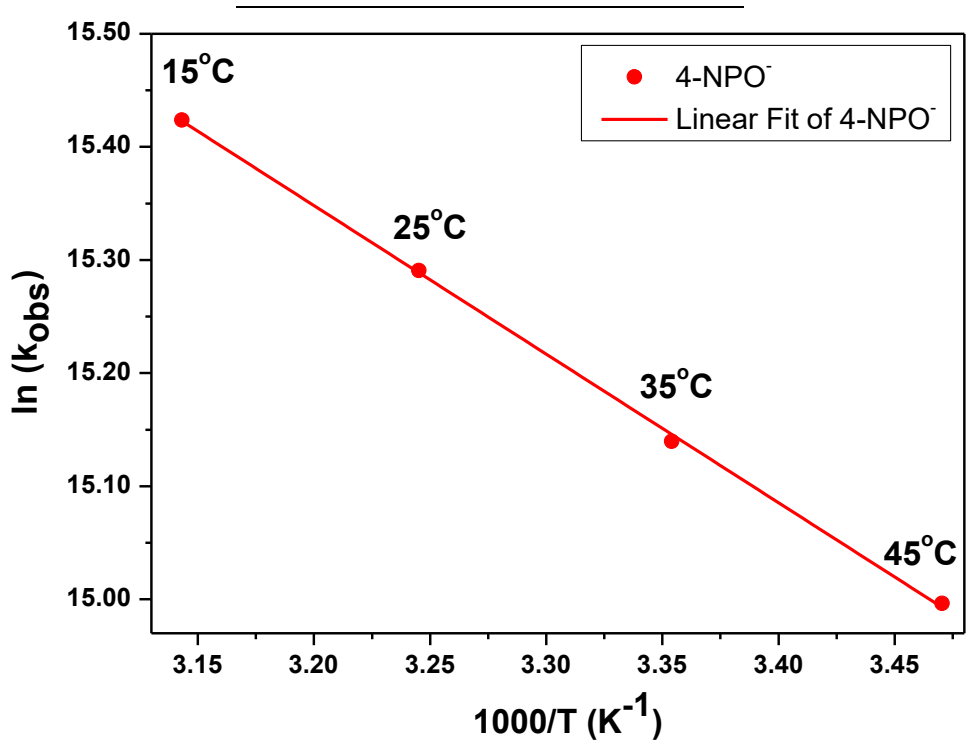


FIGURE S1 Arrhenius plot on proton transfer to 4-NPO- (red line).

Figure S2 shows linear fit for the activation parameters for 4-NPO- and shows the activation enthalpy $\left(\Delta \mathrm{H}^{\ddagger}\right)$, entropy $\left(\Delta \mathrm{S}^{\ddagger}\right)$ and Gibbs free energy $\left(\Delta \mathrm{G}^{\ddagger}\right)$ at $25^{\circ} \mathrm{C}$.

$$
\begin{gathered}
k_{o b s}=\left(k_{B} T / h\right) e^{-\left(\Delta \mathrm{H}^{\ddagger} / R T\right)} \cdot e^{\left(\Delta \mathrm{S}^{\ddagger} / R\right)} \\
\Delta G^{\ddagger}=\Delta H^{\ddagger}-T \Delta S^{\ddagger}
\end{gathered}
$$

$\Delta \mathbf{H}^{\ddagger} \quad \Delta \mathbf{S}^{\ddagger} \quad \Delta \mathbf{G}^{\ddagger}$

\section{$\left(\mathrm{kcal} . \mathrm{mol}^{-1}\right) \quad\left(\mathrm{cal}^{\mathrm{mol}} \mathrm{mo}^{-1} . \mathrm{K}^{-1}\right) \quad\left(\mathrm{kcal}^{\mathrm{mol}} \mathrm{l}^{-1}\right)$}

$2.01 \quad-21.7 \quad 8.48$

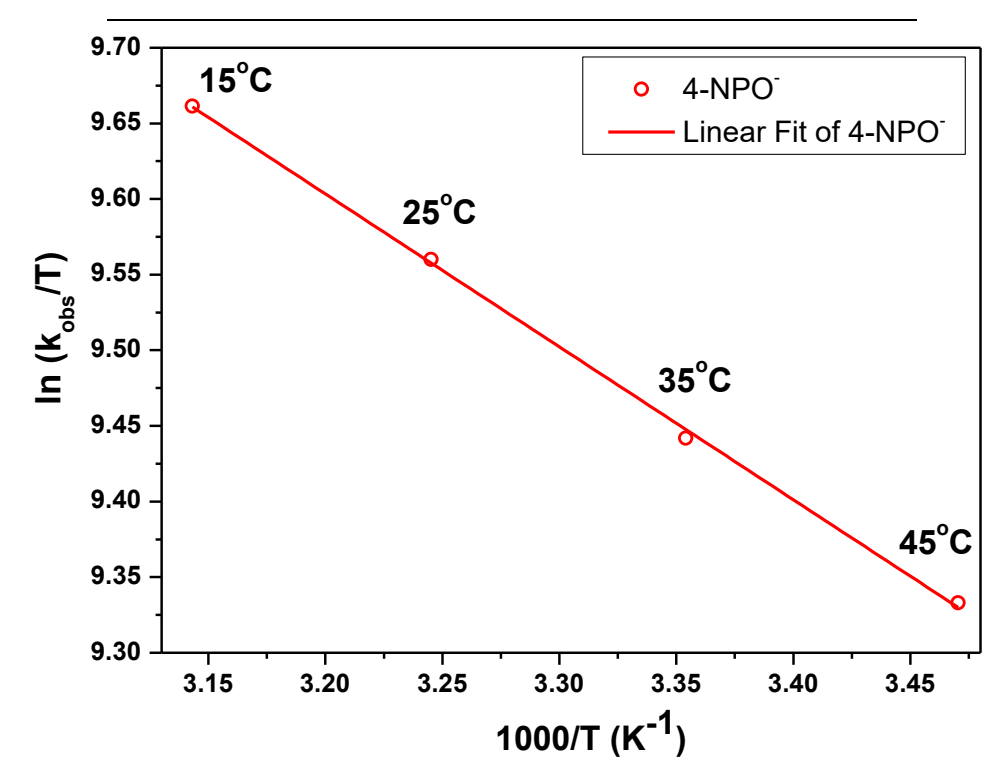

FIGURE S2 Effect of temperature on proton transfer to 4-NPO- (red line).

\section{References}

[1] E. B. Krissinel', N. Agmon, J. Comp. Chem. 1996, 17, 1085-1098.

[2] S. R. Logan, in Fundamentals of Chemical Kinetics, Longman House, 1996, pp. 264. 